\title{
Design and analysis of Lower Limb Rehabilitation Exoskeleton knee joint actuator
}

\author{
Chen Yuliang ${ }^{1}$,Cao Heng ${ }^{1}$,Zhu Jun ${ }^{1}$,Mo Songhai ${ }^{1}$ \\ 1.School of mechanical and dynamic engineering, East China University of Science and Technology \\ Shanghai, China \\ 18818206043@163.com,hengcao@163.com,jzhu_ecust@163.com,1305793251@qq.com
}

\begin{abstract}
In order to obtain a knee joint actuator with small volume, light weight and easy control, we analyzed the advantages and disadvantages of the knee joint actuators, the motion laws and motion forms of the human knee joints. We designed an exoskeleton knee actuator actuated by parallel four bar linkage. The motion of the knee joint of the exoskeleton adopts parallel four-bar linkage to ensure the linear motion of the knee joint while reducing the volume of the whole actuator. In this paper, we analyzed the kinematics of parallel four-bar linkage, and the motion characteristics of the mechanism, simulated the whole knee actuator, drew a diagram of the relation between the rotation angle of the knee joint, angular acceleration and screw displacement.
\end{abstract}

Keywords-Exoskeleton; Parallel four-bar linkage; Knee; ADAMS simulation

\section{INTRODUCTION}

Recently, the number of patients with hypertension, cardiovascular and cerebrovascular diseases is increasing, and the incidence of cardiovascular and cerebrovascular diseases is also increasing at a steady pace. According to incomplete statistics, there are as many as 250 cases of cardiovascular and cerebrovascular diseases in our country every year. About $80 \%$ of them have left different degrees of limb dyskinesia after treatment, mainly manifested as limited limb movement, atrophy and so on. By the end of 2017, there were 80 million 500 thousand disabled family workers in China, and the total number of disabled people was nearly 95 million. The emergence of the rehabilitative exoskeleton has helped many disabled people stand up again.

The earliest exoskeleton was the prototype called "Hardiman" developed by General Company in $1960^{[1] 1-2}$. In
2000, the Bleex lower limb weight-bearing exoskeleton robot appeared $^{[2] 2-3}$ Amami Kanou team of University developed HAL lower limb exoskeleton to help patients with gait disorders to walk ${ }^{[1] 4-5}$. The "InteHigent Walking Assistive Robot" of Sogang University helps the disabled and the elderly who walk inconveniently ${ }^{[1] 5-6}$. The "Lokoomat" lower extremity exoskeleton mainly trains patients with lower extremity injury, dysfunction and walking inconvenience which developed by the Federal Institute of Technology in Zurich, Switzerland, and the Balgrist Medical Rehabilitation Center ${ }^{[1] 8-9}$. In 2010, Elegs developed by Berkeley Biomimetic Technologies Inc. helped paraplegics get rid of wheelchairs completely and walk independently ${ }^{[7][8]}$. Wearable lower extremity exoskeleton robot designed by Yang Canjun team of Zhejiang University can help stroke patients perform some simple indoor rehabilitation training ${ }^{[1] 11-12}$. Fourier $X 1$, the first lower extremity exoskeleton robot launched by Fourier in 2017. At the same time, Fourier $X 1$ is the first commercial robot in China, which can be intelligently adjusted by users.

However, there are some common problems in the knee actuators of rehabilitative lower limb exoskeletons in China. For example, the knee joint actuator of the lower extremity exoskeleton robot developed by Yang Canjun team of Zhejiang University, which is driven by a common push rod mechanism, motor, through the ball screw, keep the nuts on the ball screw fixed, and the motion of the screw promotes the knee joint movement. However, its shortcomings are that the structure of the actuator is large, the motion is not smooth, the effect of force transmission is not good, and the linearity of linear motion and knee joint rotation is poor. The knee joint actuators of the wearable intelligent power assisted robot 
developed by Hefei intelligent are driven by hydraulics. The disadvantage is that the motion will be unstable when the load changes greatly, the transmission efficiency is low, and it is easy to leak.

Therefore, it is necessary to develop a rehabilitation knee joint actuator with compact structure, smooth movement, high transmission efficiency, cleanliness and easy control.

\section{MOTION ANALYSIS OF KNEE JOINT}

The knee joint is consisted of the medial and lateral femoral condyles, the medial and lateral tibial condyles and the patella. It is the largest and most complex joint in the human body and suffers more chance of injury. It belongs to the trochlear joint. The basic movement of the knee joint is flexion / extension motion, and the angle of active motion is 0 degrees $\sim 140$ degrees $^{[2] 10-12}$. In the range of motion cycle of walking, knee joint does not have a complete stretching process, and the maximum need for flexion is about 70 degrees. When the knee joint is fully straightened, the tibial intercondylar eminence is locked with the femoral intercondylar fossa, and the lateral collateral ligament is tense. Besides flexion and extension, the femoral and tibial joints can not complete other movements. When the knee joint flexes, the posterior part of both femoral condyles enters the articular fossa, the interlocking factor is relieved, the collateral ligament is relaxed, and the femoral-tibial joint can rotate slightly around the vertical axis. During knee joint movement, the meniscus can move backward when bending the knee and forward when stretching the knee. When crus rotates, the meniscus moves forward with the femoral condyle, one side slides forward and the other side slides backward.

\section{MECHANICAL STRUCTURE DESIGN}

\section{A. Selection of Driving Mode}

In the aspect of driving, not only ingenious design is needed to make the exoskeleton robot wear on human body as easy as possible without interference, but also sufficient driving force is required to achieve energy enhancement. This requires small size and large output of the driver. At present, the main driving modes are hydraulic drive, pneumatic drive and motor drive.

Hydraulic drive has good dynamic performance.
Under the same output power, the transmission process will not cause a greater impact on the mechanism, and has a certain overload protection function ${ }^{[3]}$. Its shortcomings are also prominent. Firstly, hydraulic oil is easily ignited, leaked and polluted. In many cases, there is no finished product, so it needs complex structural design, which takes a long time. The transmission efficiency is very low, its transmission accuracy is not high and the noise is high ${ }^{[9]}$.

Pneumatic drive is driven by air and other gases as energy transmission medium. It has many similarities with hydraulic drive, but also has some advantages that hydraulic drive does not have. It has low cost, green and pollution-free, little resistance in operation, high safety, and is not disturbed by high temperature. The disadvantage is that the gas density varies greatly, so the transmission stability is poor, and it is not easy to carry out accurate position control.

Motor drive is the most common way, simple principle, good motor control performance, running noise is very small, motion accuracy is high, clean, these are very suitable for rehabilitation medical equipment. At present, the control theory of motor is very mature, which can realize the complex control of exoskeleton function ${ }^{[2] 27-30}$. The encoder on the motor can output the motion state of the motor output shaft in real time, so the signal detection and processing in the process of motor driving is very convenient. Therefore, from all aspects of consideration, the final decision is to adopt motor driven mode.

\section{B. Mechanical structure of knee joint actuator}

Knee joint is one of the joints with heavy load in daily life. It is indispensable for walking, sitting, lying, running and jumping in daily life. Every pound of weight gain, the knee area has to bear six times the weight, so the protection of the knee joint is necessary. In the rehabilitative lower extremity exoskeleton (as shown in Figure 1), the knee and hip joints are driven by a parallel four-bar linkage, and the principle is roughly the same. But knee actuators are more complex and representative, so it is very important to analyze knee actuators. 


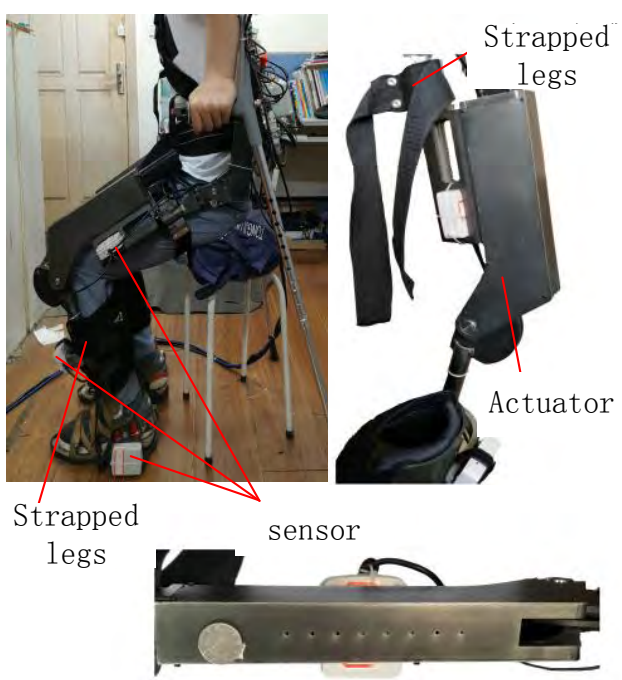

Fig.1. Rehabilitation of lower limb exoskeleton

The degree of freedom of the single knee actuator is 1 , and the actuator moves in the sagittal plane of the human body. Therefore, the requirement of exoskeleton design in this paper is that the range of DOF meets the requirements of walking and sitting in sagittal plane ${ }^{[5][6]}$. The joint torque meets the walking torque requirement after weight reduction. The actuator structure is flexible, and the structure is light. The actuator structure is protected by limited position in the limit position, which can avoid two times of injury. The prototype of the knee joint actuator and the explosion map are shown in Figure 2. The main parameters are shown in TABLE I .

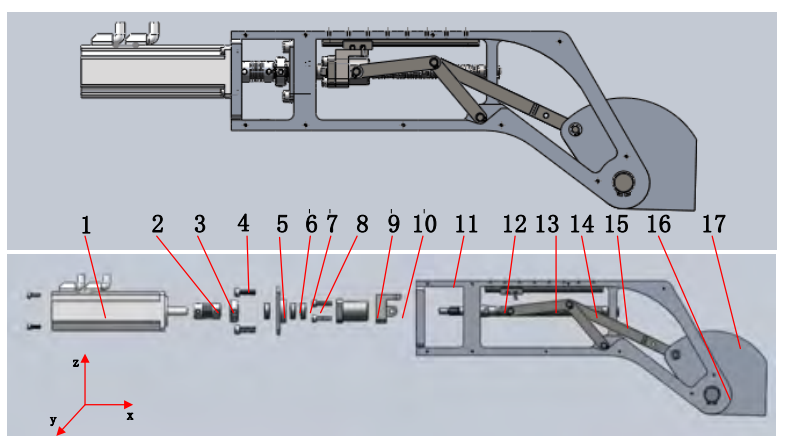

Fig.2. Knee joint actuator prototype and explosion map

In Fig. 2, 1 the motor, 2 the coupling, 3 the locking nut, 4 the bolt, 5,7 and 8 the thrust bearings, 6 bearing covers, 9 the nut of the ball screw, 10 the nut sleeve, 11 the knee joint actuator frame, 12 the ball screw, 13, 14, 15 parallel four-bar connecting rods, 16 the knee joint shaft, 17 the knee joint rotating Block. In addition to the material of parallel four connecting rods is stainless steel, the remaining structural materials are 7075 alloy aluminum.

\begin{tabular}{c|c|c}
\multicolumn{3}{c}{ TABLE I Knee joint actuator parameters } \\
\hline 1 & $\begin{array}{c}\text { Knee joint actuator } \\
\text { parameters }\end{array}$ & Technical indicators \\
\hline 2 & Motor power ( W) & 100 \\
\hline 3 & supply voltage (V) & 24 \\
\hline 4 & Total mass ( kg) & $0.8 \mathrm{~kg})$ \\
\hline 5 & Length- $\mathrm{x}(\mathrm{mm})$ & 470 \\
\hline 6 & Wide- $\mathrm{y}(\mathrm{mm})$ & Motor part 40, other 36 \\
\hline 7 & Height $-\mathrm{z}(\mathrm{mm})$ & 136 \\
\hline 8 & Range of motion of knee & $0 \sim 90^{\circ}$ \\
\hline
\end{tabular}

The knee actuator consists of a DC motor, a coupling, a ball screw and a parallel four-bar linkage. The driving scheme of the knee joint actuator is shown in Fig. 3. The rotation of the motor drives the ball screw to rotate through the coupling. The nut on the ball screw moves in a straight line along the ball screw, and the knee joint is driven by the parallel four-bar linkage mechanism.

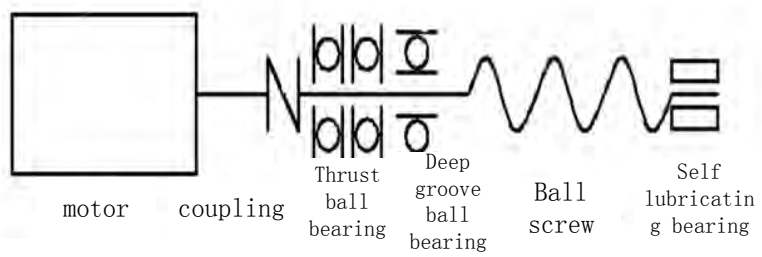

Fig.3. Actuator power drive schematic diagram

In order to reduce the vibration during the movement of ball screw and make the force and moment transfer stable and reliable, three bearings ${ }^{[10] 3-280}$ (as shown in Fig. 3) are used to support the ball screw structure. At the same time, the axial force and the radial force are larger, which can reduce the processing accuracy of the bearing seat and reduce the cost. 


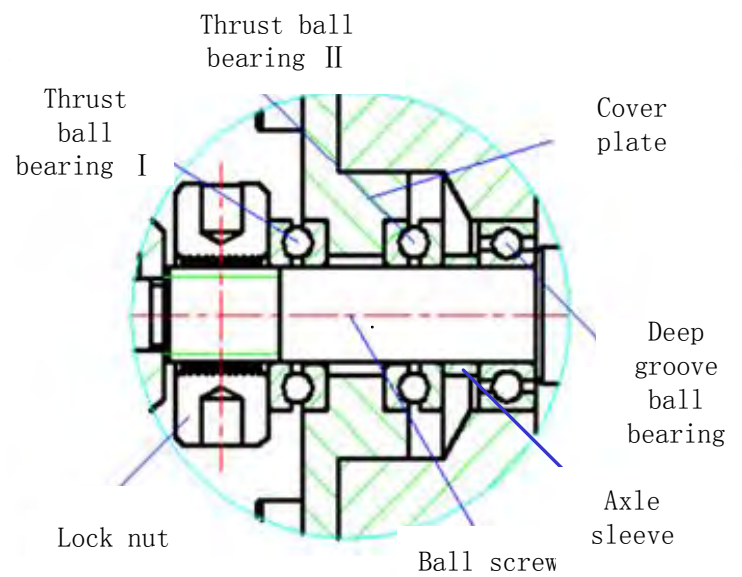

Fig.4. Bearing installation drawing length of $\operatorname{rod} B C$ and $\operatorname{rod} D E$ is $l_{2}$.

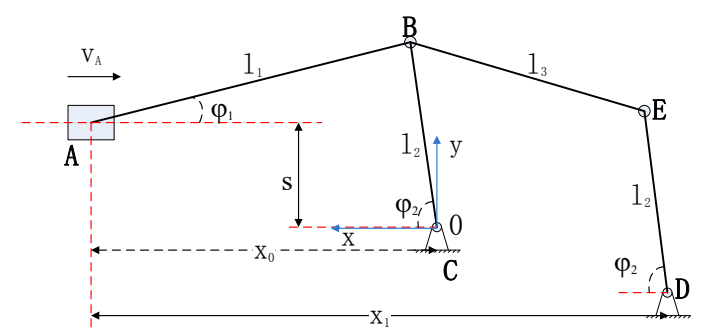

Fig.5. Parallel four-bar linkage

\section{B. kinematic analysis}

The origin of coordinates is set at the intersection point between the horizontal line of $A$ and the vertical line of $C$ (as shown in Fig.5). Then we can get the dynamic position expression of point $A$.

$$
x_{A}=x_{0}-v_{A} \cdot t
$$

Where, $x_{0}$ is the initial position of nut $A$, and $v_{A}$ is the speed of point $A$. Because the nut $A$ is driven by the motor through the coupling and lead screw. Then, we have the following expressions:

$$
v_{A}=n_{0} \cdot h
$$

$n_{0}$ is the speed of the motor and $h$ is the lead of the ball screw.

$$
\begin{aligned}
& l_{1} \cos \varphi_{1}+l_{2} \cos \varphi_{2}=x_{\mathrm{A}} \\
& l_{1} \sin \varphi_{1}+s=l_{2} \sin \varphi_{2}
\end{aligned}
$$

The formula (3) and the formula (4) can be obtained separately:

$$
\begin{aligned}
& \cos \varphi_{1}=\frac{x_{A}-l_{2} \cos \varphi_{2}}{l_{1}} \\
& \sin \varphi_{1}=\frac{l_{2} \sin \varphi_{2}-s}{l_{1}}
\end{aligned}
$$

because $\cos ^{2} \varphi_{1}+\sin ^{2} \varphi_{1}=1$, we can obtain the following equation:

$$
x_{A}{ }^{2}+s^{2}+l_{2}{ }^{2}-l_{1}{ }^{2}=2 l_{2}\left(x_{A} \cos \varphi_{2}+s \sin \varphi_{2}\right)
$$

Assume $\sin \alpha=\frac{x_{A}}{\sqrt{x_{\mathrm{A}}^{2}+s^{2}}}, \cos \alpha=\frac{s}{\sqrt{x_{\mathrm{A}}^{2}+s^{2}}}$, The formula

(7) becomes the following:

$$
\begin{aligned}
\sin \alpha \cos \varphi_{2}+ & \cos \alpha \sin \varphi_{2}=\frac{x_{A}{ }^{2}+s^{2}+l_{2}{ }^{2}-l_{1}{ }^{2}}{2 l_{2} \sqrt{x_{\mathrm{A}}{ }^{2}+s^{2}}} \\
\sin \left(\alpha+\varphi_{2}\right) & =\frac{x_{A}{ }^{2}+s^{2}+l_{2}{ }^{2}-l_{1}{ }^{2}}{2 l_{2} \sqrt{x_{\mathrm{A}}{ }^{2}+s^{2}}}
\end{aligned}
$$




$$
\varphi_{2}=\pi-\sin ^{-1} \frac{x_{A}^{2}+s^{2}+l_{2}^{2}-l_{1}^{2}}{2 l_{2} \sqrt{x_{\mathrm{A}}{ }^{2}+s^{2}}}-\sin ^{-1} \frac{x_{A}}{\sqrt{x_{\mathrm{A}}^{2}+s^{2}}}
$$

The relationship between the speed of the nut movement and the rotation angle of the knee can be obtained through the formula (1) and the formula (9).

\section{ADAMS SIMULATION ANALYSIS}

The three-dimensional Solidworks model of the Rehabilitation Exoskeleton knee joint actuator is saved as the corresponding format and imported into ADAMS software. The material characteristic parameters of each part of the model are set according to the actual material. By adding appropriate constraints and setting the appropriate speed of the motor, the motion curve of the knee joint actuator is close to that of the disabled. The lead of the ball screw is $2 \mathrm{~mm}$, the model set up is shown in Fig. 6.

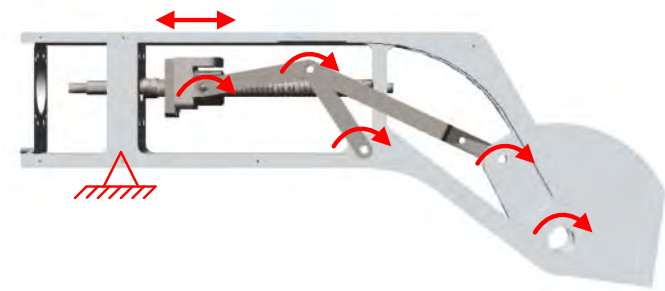

Fig.6. Knee actuator model in ADAMS

The driving input of the model is the input according to the walking cycle of the disabled. Because during normal walking, the rotation angle of the knee does not exceed 70 degrees. So the function of input speed of motor in ADAMS is as follows:STEP5(time, $0,0 d, 1,-2000 d)+S T E P 5($ time , 1 , Od , 4 , 1500d )+STEP5( time , 4 , Od, 7.5 , -10000d )+STEP5( time, 7.5, Od, 10, 10500d $)$, The cycle is $10 \mathrm{~s}^{[2] 15-17}$. Using ADAMS software post-processing module to simulate and analyze the knee joint actuator. We can obtain the curves of rotation angle, angular velocity, angular acceleration of unilateral knee joint actuator and displacement, velocity and acceleration of screw nut, as shown in figs. 7, 8 and 9. The red solid line in the picture is the curve of the knee joint, and the blue dotted line is the curve of the nut.

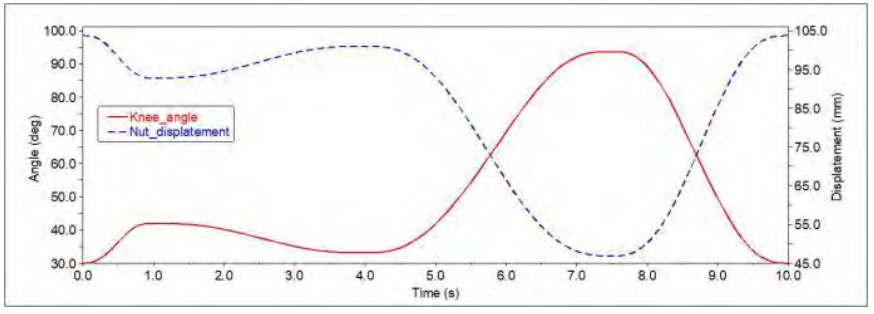

Fig.7. Knee joint actuator rotation angle and screw nut displacement

From figs. 7, 8 and 9, we can be seen that the curve of knee joint rotation angle and screw nut displacement is almost the same, and the linearity is very good. So this provides a very good condition for controlling knee joint motion, that is, controlling the movement of nuts can control the knee joint angular motion. The angular velocity of the knee joint and the speed of the screw nut, the angular acceleration of the knee joint and the acceleration of the screw nut are relatively smooth, which will not make the users feel uncomfortable.

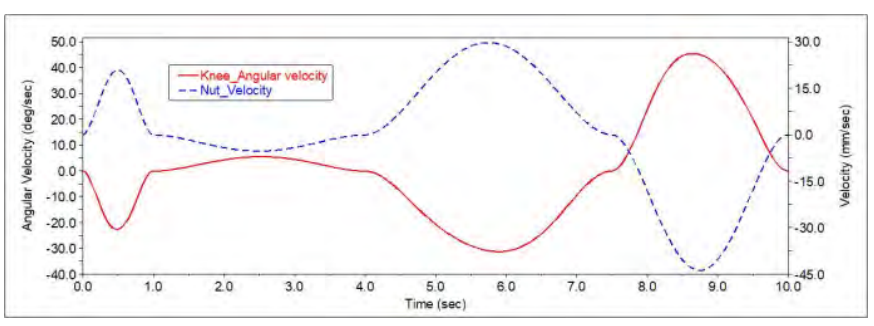

Fig.8. Knee angular velocity and lead screw velocity

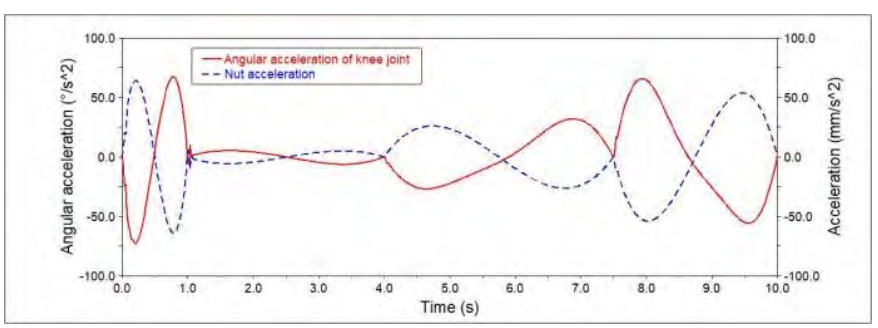

Fig.9. Knee angular acceleration and screw acceleration

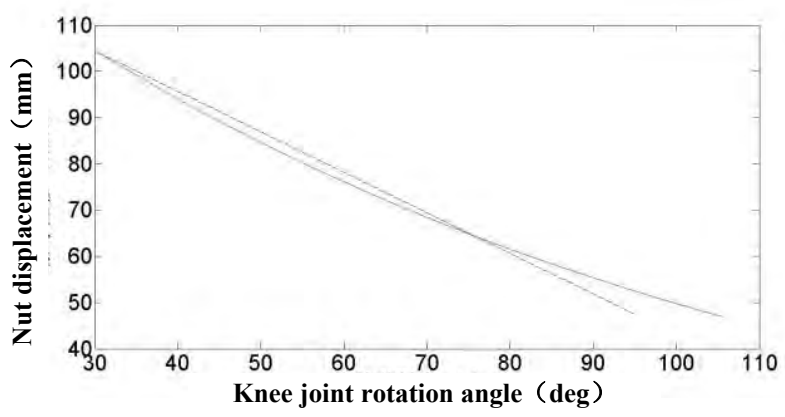

Fig.10. Relationship between rotation angle of knee joint and screw nut displacement

The relationship between the rotation angle of the knee 
and the displacement of the screw nut is shown in Fig.10. They have good linearity (solid lines represent the relationship between them, dashed lines represent the closest oblique line of the curve). This shows that the knee joint motion angle can be controlled accurately only by controlling the forward and backward rotation of the motor and the rotational speed of the motor. The control accuracy is effectively improved, and the basis for further control is further established.

\section{CONCLUSION}

In this paper, we present a design of a rehabilitation knee joint actuator for lower limb exoskeleton based on parallel four-bar linkage. It can help the knee joint flexion / extension movement of the lower extremity disabled people. The actuator ball screw is driven by DC motor to facilitate subsequent control. The kinematics analysis of the parallel four-bar linkage is carried out, and we obtain the relationship between the rotation angle of the linkage and the displacement of the ball screw nut. Using ADAMS software to simulate the flexion/extension motion of the knee joint actuator of the lower extremity exoskeleton, the linear relationship between the motor speed and the knee joint rotation angle was further validated. We obtain the relationship between the displacement, velocity and acceleration of the screw nut and the rotation angle, angular velocity and angular acceleration of the knee joint. It provides a basis for controlling the knee joint of rehabilitation lower limb exoskeleton.

\section{ACKNOWLEDGMENT}

This work was supported by the National Natural Science Foundation of China under Grant No. 91748110.

\section{REFERENCES}

[1] Jiashan. Kinetic analysis and motion planning of lower extremity exoskeletons [D]. Southeast University, 2016:1-116

[2] Chen Wei. Structural design and reliability analysis of bionic lower extremity exoskeleton robot [D]. University of Electronic Science and Technology, 2015:9-41

[3] Pei Xiang, Li Qucheng, Jin Dingcan. Design and analysis of a series three-degree-of-freedom hip joint mechanism [J]. Mechanical design and research, 2014:51-54.

[4] Shi Xiaohua, Wang Hongbo, Sun Li. Structural design and dynamic analysis of exoskeleton lower limb rehabilitation robot $[\mathrm{J}]$. Journal of Mechanical Engineering, 2014 (03): 47-54.

[5] Fang Mingzhou, Wang Yu, Zhu Jun. Research and Simulation of heavy-duty exoskeleton robot mechanism of lower limbs [J]. Journal of East China University of Technology (Natural Science Edition), 2014:118-121.

[6] Rao Lingjun, Xie Wei, Zhu Xiaobiao. Research and design of lower extremity exoskeleton walking rehabilitation robot $[\mathrm{J}]$. Mechanical design and research, 2012 (03): 31-33.

[7] Huang R., Cheng H, Chen Q., et al. Interactive learning for sensitivity factors of a human-powered augmentation lower exoskeleton.Ieee/rsj International Conference on Intelligent Robots and Systems. IEEE, 2015:6409-6415

[8] Huang R., Cheng H., Guo H., et al. Hierarchical Interactive Learning for a HUman-Powered Augmentation Lower Exoskeleton.IEEE International Conference on Robotics and Automation. IEEE, 2016:257-263

[9] Zheng Jianrong. Introduction and improvement of ADAMS virtual prototyping technology. Beijing: Machinery Industry Press, 2001:93-185.

[10] Cheng Daxian. Editor-in-chief. Mechanical Design Manual. Fourth Edition. Beijing. Chemical Industry Publishing House, 2002:3-467. 